\title{
RFLP-PCR is more efficient than ARMS-PCR for identifying CYP2C19*2 polymorphism in atherosclerotic patients
}

\author{
I.R. Costa ${ }^{1,2,3}$, T.R. Santos ${ }^{1,2}$, I.A. Bento ${ }^{1,2}$, B.O. Siqueira ${ }^{1,2}$, A.M. Barbosa ${ }^{1,3}$, \\ K.S.F. Silva ${ }^{1,3}$, M. H. Lagares ${ }^{1}$, J.V.M. Martins ${ }^{1}$, D.A. Rodrigues ${ }^{1}$, \\ M.P. Morais ${ }^{1}$, F.L. Campedelle ${ }^{1}$ and K.K.V.O. Moura ${ }^{1,2}$ \\ ${ }^{1}$ Núcleo de Pesquisas Replicon - Pontifícia Universidade Católica de Goiás, \\ Goiânia, GO, Brasil \\ ${ }^{2}$ Escola de Ciências Médicas, Farmacêuticas e Biomédicas - Pontifícia \\ Universidade Católica de Goiás, Goiânia, GO, Brasil \\ ${ }^{3}$ Universidade Federal de Goiás, Goiânia, GO, Brasil \\ Corresponding author: K.S.F. Silva \\ E-mail: smallbinho@hotmail.com \\ Genet. Mol. Res. 18 (3): gmr18268 \\ Received February 22, 2018 \\ Accepted July 01, 2019 \\ Published July 12, 2019 \\ DOI http://dx.doi.org/10.4238/gmr18268
}

\begin{abstract}
The CYP2C19*2 polymorphism is the result of a point mutation leading to an alternative splicing defect, generating an enzyme with a reduced function. The enzyme CYP2C19 is responsible for the activation of the prodrug Clopidogrel and the loss of its function leads to an increase in platelet aggregation. We compared the ARMS-PCR (Amplification Refraction Mutation System) and RFLP-PCR (Restriction Fragment Length Polymorphism) to determine which of the techniques was more efficient for identifying the CYP2C19*2 polymorphism. We analyzed 200 samples from patients with atherosclerosis and 100 samples from a control group, free from the disease. The polymorphic allele was not observed in any of the samples analyzed by the ARMSPCR technique, but was present in more than $70 \%$ of the samples when analyzed by RFLP-PCR. Thus, RFLP-PCR generated more satisfactory results for the analysis of CYP2C19*2 polymorphism, and it should be used, despite the lower cost of ARMS-PCR, as the latter did not detect this polymorphism.
\end{abstract}

Key words: RFLP-PCR; ARMS-PCR; CYP2C19*2 polymorphism; Atherosclerosis 


\section{INTRODUCTION}

CYP2C19 is a liver enzyme responsible for catalyzing bioactivation of the antiplatelet prodrug Clopidogrel; CYP2C19 loss of function increases the risk for serious cardiovascular events, such as acute coronary syndromes. The wild allele $* 1$ is associated with functional metabolism of the CYP2C19 gene (Scott et al., 2013). The antiplatelet activity of Clopidogrel may be decreased or even absent in individuals carrying allele $* 2$ (Santos et al., 2014). The CYP2C19*2 (G681A) polymorphism consists of a point mutation, where a guanine is replaced by an adenine at position 681 of exon 5 of the CYP2C19 gene. This SNP leads to an alteration of alternative splicing and the production of an inactive protein (Santos et al., 2014; Denisenko et al., 2017). Sibbing et al. (2010) showed a relation between alleles $* 2$ and $* 17$. They observed a gradual increase in platelet aggregation values in the presence of the $* 2$ allele, but that aggregation is reduced with the concomitant presence of the allele $* 17$.

Another polymorphism, CYP2C19*3 (G636A), occurs when there is a change of a guanine for an adenine at position 636 of exon 4. This SNP leads to the formation of a stop codon and the production of an inactive protein (Santos et al., 2014). Allele *2, CYP2C19*3 is also responsible for a decrease in or absence of CYP2C19 activity. However, it has a lower frequency, with less than $1 \%$ in Caucasians and may vary from 2 to 9\% in Asians (Scott et al., 2013; Denisenko et al., 2017).

The cost of most PCR techniques is relatively high; however, there are low cost systems that involve a single PCR reaction, followed by electrophoresis in agarose or polyacrylamide gel, such as ARMS-PCR (Amplification Refraction Mutation System), which makes the technique more accessible (Gandra et al., 2008; Guimarães et al., 2009). ARMS-PCR differs from an RFLP-PCR for allele determination since it does not require the use of a restriction enzyme in order to cleave the amplified DNA. We compared the ARMS-PCR and RFLP-PCR techniques for the analysis of the CYP2C19*2 polymorphism in order to determine which is more efficient in the detection of this polymorphism and which presents the best cost-benefit ratio for this detection.

\section{MATERIAL AND METHODS}

We analyzed 200 samples from patients with atherosclerosis and 100 samples from a control group, free from the disease. DNA samples from patients with and without atherosclerosis were previously submitted to molecular tests with ARMS-PCR at the Replicon Laboratory in Pontifical University Catholic of Goiás (PUC-GO), in order to detect the CYP2C19*2 polymorphism. We analyzed 300 samples, which were divided into two groups: case (patients with atherosclerosis) and control (patients without atherosclerosis). PCR reactions were performed according to the protocol proposed by Sambrook et al. (2001) with a final volume of $25 \mu \mathrm{L}$. The PCR-RFLP technique was used to confirm the CYP2C19*2 polymorphism and define which of the techniques is most costeffective. ARMS-PCR, although less expensive, did not present satisfactory results in the detection of CYP2C19*2 polymorphisms in previous studies (Guimarães et al., 2009). The project was approved by the Research Ethics Committee of PUC Goiás according to protocol no. 35321614.3.0000.0037 and the participants signed informed consent forms. The authors state that there is no conflict of interest. 


\section{RESULTS}

It has been shown that CYP2C19*2 polymorphisms are related to atherosclerosis in certain ethnic groups, including the Brazilian population. It has also been demonstrated that different polymorphism identification techniques show different sensitivity. Thus, we decided to analyze the presence of CYP2C19*2 polymorphism in a population in CentralWest Brazil by two different techniques in order to decide which is more effective and with the lowest cost-benefit. The presence of at least one polymorphic allele of the CYP2C19*2 gene was detected in $74.5 \%$ of 200 patients with atherosclerosis by the PCR-RFLP technique. No polymorphic alleles of the CYP2C19*2 gene were detected by the ARMSPCR technique. In the control group, through the RFLP technique, the presence of at least one polymorphic allele of the CYP2C19*2 gene was detected in $73 \%$ of the 100 patients. No polymorphic alleles of the CYP2C19*2 gene were detected by the ARMS technique in the case or control group.

\section{DISCUSSIONS}

Cardiovascular diseases have a multifactorial character and bring consequences not only for the carrier but also for the entire health system, being one of the biggest public health problems in Brazil and worldwide. In this regard, more and more researchers try to address this problem. The use of pharmacogenetics attempts to obtain better results and fewer side effects for cardiovascular treatment (Shin et al., 2012). Patients present variable responses to drugs, both in terms of their positive effects and their toxicity, which makes even more important the use of molecular biology in the analysis of patients who are under treatment (Metzger et al., 2006).

Molecular markers are widely used and are characterized by the detection of genetically inherited natural variations in DNA sequences between individuals (Ye et al., 2001). In addition to targeted genotyping, laboratories can test CYP2C19 through direct sequencing and clinical sequencing programs for the entire exome and genome are increasingly being applied in academic medical centers and even in commercial laboratories. Sequence-based tests are able to identify common variants of CYP2C19 $(* 2$ and $* 3$ for example), but also identify rare alleles $(* 4$ and $* 8$ for example) and novel CYP2C19 variant alleles that have no proven clinical significance as a response to Clopidogrel (Scott et al., 2013).

Clopidogrel requires its conversion into its active metabolite through hepatic mechanisms involving a large amount of CYP450 isoenzymes, among them CYP2C19, responsible for almost half of the phase I detoxification processes (Marchini et al., 2017). At least three alleles have been detected for CYP2C19 isoenzyme and two of them are associated with a loss of function: CYP2C19*2 and *3. These variants comprise from $85 \%$ to $99 \%$ of the alleles that cause loss of response to Clopidogrel especially in Caucasians and Asians. Since 2010, after an FDA warning, scientific interest has increased regarding studies that could determine individuals' lack of response to Clopidogrel (Santos et al., 2014). The polymorphism CYP2C19 *2, shows an approximate frequency of $15 \%$ in Caucasians and can reach $35 \%$ in Asians, leading to a high rate of loss of gene function (Scott et al., 2013) within those populations. In addition, except for Asians the frequency of 
the CYP2C19*2 polymorphism is around $15 \%$, for example it was found to be $18 \%$ in Mexicans and 15.5\% in Brazilians (Favela-Mendoza et al., 2015).

The ARMS-PCR system is performed by combining two allele-specific primers and two common external primers. The allelic specificity is conferred by the difference between the base at the 3' end of the internal primers and the base of the alternative allele of the SNP. As in the ARMS system, the design of compatible primers for simultaneous amplification is often challenging, it increases the probability of errors, making the technique more limited in relation to RFLP (Guimarães et al., 2009). Variations detected by the RFLP technique occur due to simple mutations, rearrangements of the DNA segments by deletions, insertions or translocations and recombination, which may occasionally alter the sequence of one or more recognition sites of a given enzyme (Ye et al., 2001).

Duta-Cornescu et al. (2009) compared the ARMS and RFLP techniques for the identification of SNPs. They concluded that the ARMS system would be more advantageous, a fast, reliable and non-isotopic method, in which the results can be easily obtained in one working day. The RFLP system is more handful, requiring optimization steps, which increases the cost-effectiveness of the former technique. The ARMS system is faster and easier to perform. However, the technique did not present reliable results in the present work, while the RFLP technique identified at least a polymorphic allele in more than $70 \%$ of the patients, whereas the ARMS technique identified no polymorphism in any patient (Duta-Cornescu et al., 2009).

The inability of the ARMS-PCR technique to identify the CYP2C19*2 polymorphism shows the inefficacy of the procedure, it is known that the CYP2C19*2 polymorphism is present in at least $15 \%$ of the population. The most common causes for the inefficacy of the technique are hairpin loop formation, annealing with the other primer (primer dimer) and dramatic difference in annealing temperature of each primer, which should not exceed $5^{\circ} \mathrm{C}$. In the present research, three sets of different primers were used in order to detect the CYP2C19*2 polymorphism. However, ARMS-PCR was unable to identify the amplification of the polymorphic allele in any of the presented situations, except for the control with the wild allele. Different concentrations of $\mathrm{MgCl}_{2}$ and a temperature gradient were performed in order to identify the ideal hybridization temperature of the primers but even so, the polymorphism was not identified by the ARMS-PCR technique. The primers were drawn from tools present on the NCBI website carefully not to induce hairpin and dimer formation (Lorenz, 2012; Scott et al., 2013).

According to Hirata et al. (2006), the specificity of the ARMS-PCR reaction is influenced by variations in reagent concentrations, temperature, hybridization time and number of polymerization cycles. The addition of formamide may reduce the generation of non-specific products. Under conditions of low stringency (when the primer binds unspecifically) hybridization and primer extension occur in the sequences of both alleles, leading to incorrect results. When the frequency of the mutant allele is low, the specificity of the technique becomes critical and the possibility of false-positive or negative results shows the limitation of the technique.

\section{CONCLUSIONS}

We conclude that the RFLP-PCR technique generated the best results. ARMS-PCR failed to identify polymorphic alleles in many patients, which makes clear the need for a 
more sensitive technique such as RFLP. Although the latter has a significantly higher cost, the results are safer, so it is recommended to use restriction enzymes to confirm the identification.

\section{REFERENCES}

Denisenko NP, Sychev DA, Sizova ZM, Ryzhikova KA, et al. (2017). Urine metabolic ratio of omeprazole in relation to CYP2C19 polymorphisms in Russian peptic ulcer patients. Pharmgenomics Pers. Med. 10: 253-9.

Duta-Cornescu G, Simon-Gruita A, Constantin N, Stanciu F, et al. (2009). A comparative study of ARMS - PCR and RFLP - PCR as methods for rapid SNP identification. Rom. Biotechnol. Lett. 14(6): 4845-50.

Favela-Mendoza AF, Martinez-Cortes G, Hernandez-Zaragoza M, Salazar-Flores J, et al. (2015). Genetic variability of CYP2C19 in a Mexican population: contribution to the knowledge of the inheritance pattern of CYP2C19*17 to develop the ultrarapid metabolizer phenotype. J. Genet. 94(1): 3-7.

Gandra EÁ, Gandra TKV, Mello WS and Godoi HS (2008). Técnicas moleculares aplicadas à microbiologia de alimentos. Acta Sci. Technol. 30(1): 109-18.

Guimarães CT, Magalhães JV, Lanza MA and Schuster I (2009). Marcadores moleculares e suas aplicações no melhoramento genético. Inf. Agropecuário. 30(253): 24-33.

Hirata MH, Tavares V and Hirata RDC (2006). Da biologia molecular à medicina: Métodos comumente utilizados em farmacogenética. Medicina Ribeirao Preto. 39(4): 522-34.

Lorenz TC (2012). Polymerase Chain Reaction: Basic Protocol Plus Troubleshooting and Optimization Strategies. J. Vis. Exp. 63: 1-15.

Marchini JF, Pinto MR, Novaes GC, Badran AV, et al. (2017). Decreased platelet responsiveness to clopidogrel correlates with CYP2C19 and PON1 polymorphisms in atherosclerotic patients. Braz. J. Med. Biol. Res. 50: (1) e5660.

Metzger IF, Souza-Costa DC and Tanus-Santos JE (2006). Farmacogenética: Princípios, Aplicações e Perspectivas. Medicina Ribeirao Preto. 39(4): 515-21.

Santos A, Márquez I, Arria M, Brito J, et al. (2014). Determinación del polimorfismo CYP2C19*2 en los pacientes con síndrome coronario agudo ingresados en el Hospital "Dr Domingo Luciani". Av. Cardiol. 34(1): 33-9.

Scott SA, Sangkuhl K, Stein CM, Hulot J, et al. (2013). Clinical Pharmacogenetics Implementation Consortium Guidelines for CYP2C19 Genotype and Clopidogrel Therapy : 2013 Update. Clin. Pharmacol. Ther. 94(3): 317-23.

Shin DJ, Kwon J, Park AR, Bae Y, et al. (2012). Association of CYP2C19*2 and *3 genetic variants with essential hypertension in Koreans. Yonsei Med. J. 53(6): 1113-1119.

Sibbing D, Gebhard D, Koch W, Braun S, et al. (2010). Isolated and interactive impact of common CYP2C19 genetic variants on the antiplatelet effect of chronic clopidogrel therapy. J. Thromb. Haemost. 8(8): 1685-93.

Ye S, Dhillon S, Ke X, Collins AR, et al. (2001). An efficient produce for genotyping single nucleotide polymorphisms. Nucleic Acids Res. 17(29): 1-8. 